\title{
ANOMALOUS DIFFUSION ON FRACTAL STRUCTURE OF MAGNETIC MEMBRANES*
}

\author{
Anna Strzelewicz ${ }^{\dagger}$, Monika Krasowska, Gabriela Dudek \\ Aleksandra Rybak, Roman Turczyn \\ Department of Physical Chemistry and Technology of Polymers \\ Silesian University of Technology \\ Ks. M. Strzody 9, 44-100 Gliwice, Poland
}

\section{Michae Cieśla}

The M. Smoluchowski Institute of Physics, Jagiellonian University Reymonta 4, 30-059 Kraków, Poland

(Received May 27, 2013)

The concept of diffusion on fractal structure of polymeric membrane with magnetic powder is presented. The fractal characteristics, i.e. static fractal dimension $d_{\mathrm{f}}$ and fractal dimension of the trajectory of the random walk $d_{\mathrm{w}}$, were evaluated for qualitative and quantitative description of membrane structures. The way of introducing the fractal dimension and anomalous-diffusion exponent into the generalized diffusion equation on fractal geometries obtained by Metzler et al. has been shown. The results showed that the random walk within the membranes of the smallest granulation of magnetic powder was of the most subdiffusive character.

DOI:10.5506/APhysPolB.44.955

PACS numbers: 05.45.Df, 51.10. $+\mathrm{y}, 68.55 . J \mathrm{k}$

\section{Introduction}

In recent years a great number of studies have been carried out in order to gain a better understanding of transport phenomenon in membranes with disordered structure [1-7]. The standard theories of transport poorly apply in such systems, since many physical properties (in particular the

* Presented at the XXV Marian Smoluchowski Symposium on Statistical Physics, "Fluctuation Relations in Nonequilibrium Regime", Kraków, Poland, September 10-13, 2012.

$\dagger$ Anna.Strzelewicz@polsl.pl 
diffusion) became anomalous [8]. Occasionally, even in fairly standard approach, an acceptable agreement with experimental data can be reached [9] what "proofs", in a sense, a nonuniqueness of a mathematical description in most of the interesting cases i.e. quite a few different models can represent the same, uniquely existing, solution [10]. In cases, however, when the structure of the "diffusive space" had been detected as self-similar, the concept of fractals should be applied. Fractal models may be used both, in the study of static as well as dynamic properties of disordered structures $[1,11]$. Recently [12, 13], we have discussed structure-morphology problems of magnetic membranes used to the air separation. These were polymer membranes filled with magnetic powder and magnetized. A dense polymer with added magnetic powder could be considered as disordered system because of penetrant-scale gaps whose size and position are changing randomly. Such medium shows typical stochastic geometrical characteristics and could be described within the percolation theory which is one of the tools for dealing with the random systems [1]. Furthermore, our "magnetic" membranes with molecular-scale gaps show distinctive fractal characteristics [12].

A process is referred to as anomalous diffusion if mean squared displacement of the randomly walking molecule i.e.

$$
\left\langle r^{2}(t)\right\rangle \sim t^{\alpha}
$$

deviates (with $\alpha \neq 1$ ) from the linear dependence that characterizes normal diffusion. The anomalous diffusion exponent $\alpha$ specifies the process of mass transport in the following way: if $0<\alpha<1$, the motion is called subdiffusive, if $\alpha>1$, superdiffusive [14]. The diffusion on fractals is generally anomalous and the mean square displacement of the walker scales with $t$ as

$$
\left\langle r^{2}(t)\right\rangle \sim t^{2 / d_{\mathrm{w}}},
$$

where $d_{\mathrm{w}}$ is defined as the dimension of a random walk, and its value is usually $d_{\mathrm{w}}>2$. This anomaly is due to the geometric obstacles existing on all length scales that slow down the random walker and only reduced diffusion speed occurs [15]. There is no doubt that the fractal dimension of a membrane should be taken into account while constructing relations like (2). For a certain type of fractals, such relation already exists $[1,16]$

$$
\left\langle r^{2}(t)\right\rangle \sim t^{2 /(2+\theta)},
$$

where

$$
\theta=2\left(d_{\mathrm{f}}-d_{\mathrm{s}}\right) / d_{\mathrm{s}}
$$

and

$$
d_{\mathrm{s}}=2 d_{\mathrm{f}} / d_{\mathrm{w}} .
$$


If one wants to use relations (1) and (3), one needs to know the values of $d_{\mathrm{f}}$ and $d_{\mathrm{w}}$ for a given membrane. The value of $d_{\mathrm{f}}$ can be estimated using the method of box counting, in the case where we have cross section or surface images from optical microscopy of membranes, while estimation of $d_{\mathrm{w}}$ is based on random walk simulation and relation (2). The most famous example of a fractal is the Sierpinski gasket, which has a fractal dimension $d_{\mathrm{f}}=1.58$ and dimension of random walk $d_{\mathrm{w}}=2.32$ [1]. The dynamical properties of complex systems are characterized through the probability densities of a random walker or simply of its normalized concentration, that are of general interest and of great importance. Several authors presented asymptotic probability density functions for the location of a random walker on a fractal object. Metzler et al. [2] introduced the generalized diffusion equation on fractals, where the parameters of this fractional partial differential equation are uniquely determined by the fractal Hausdorff dimension of the underlying object and the anomalous diffusion exponent. The generalized diffusion equation can be written out as follows

$$
\frac{\partial^{\frac{2}{d_{\mathrm{w}}}} P(r, t)}{\partial t^{\frac{2}{d_{\mathrm{w}}}}}=\frac{1}{r^{d_{s}-1}} \frac{\partial}{\partial r}\left(D r^{d_{s}-1} \frac{\partial P(r, t)}{\partial r}\right)
$$

The equation, compatible with condition (2), can be solved exactly in terms of Fox' $H$-function. The solution procedure with conditions, that for $r \rightarrow 0$, $P(0, t) \propto t^{-\frac{d_{\mathrm{s}}}{2}}$ and for $t \rightarrow 0, r^{d_{\mathrm{f}}-1} P(r, t)$ approaches $\delta(r)$, is shown in [2]. The authors re-scale the diffusion equation to absorb the diffusion constant. This equation (6) is a generalization of the spherically symmetric diffusion equation in Euclidean spaces, where the domain of $r$ is $[0,+\infty)$.

Such equation used with aforementioned conditions can model process of desorption as in the case of diffusive release from polymeric microspheres. The solution of Eq. (6), i.e. probability density function for a random walker on a fractal lattice, is given by

$$
\begin{aligned}
P(r, t)= & A^{*} \frac{2}{d_{\mathrm{w}}} t^{-\frac{d_{\mathrm{f}}}{d_{\mathrm{w}}}} \times\left[\sum_{j=0}^{\infty} \frac{\Gamma\left(\frac{d_{\mathrm{f}}}{d_{\mathrm{w}}}-1-j\right)}{\Gamma\left(1-\frac{d_{\mathrm{f}}}{d_{\mathrm{w}}}-\frac{2}{d_{\mathrm{w}}}\left(1-\frac{d_{\mathrm{f}}}{d_{\mathrm{w}}}+j\right)\right)}\right. \\
& \times \frac{(-1)^{j}}{j !}\left(\frac{r^{d_{\mathrm{w}}}}{t}\right)^{\frac{2}{d_{\mathrm{w}}}\left(1-\frac{d_{\mathrm{f}}}{d_{\mathrm{w}}}+j\right)} \\
& \left.+\sum_{j=0}^{\infty} \frac{\Gamma\left(1-\frac{d_{\mathrm{f}}}{d_{\mathrm{w}}}-j\right)}{\Gamma\left(1-\frac{d_{\mathrm{f}}}{d_{\mathrm{w}}}-\frac{2}{d_{\mathrm{w}}} j\right)} \frac{(-1)^{j}}{j !}\left(\frac{r^{d_{\mathrm{w}}}}{t}\right)^{\frac{2}{d_{\mathrm{w}}} j}\right],
\end{aligned}
$$


where the constant $A^{*}$ is defined as

$$
A^{*}=\frac{2^{-d_{\mathrm{w}}-3} d_{\mathrm{w}}}{\Gamma\left(\frac{1}{2}+d_{\mathrm{f}}-\frac{1}{2} d_{\mathrm{s}}\right) \Gamma\left(\frac{1}{2} d_{\mathrm{f}}\right)} .
$$

Equation (6) may be reduced to the standard diffusion equation in $d$-dimensional Euclidean space by appropriate choice of the free parameters, i.e. $d_{\mathrm{f}}=2$ and $d_{\mathrm{w}}=2[2]$.

\section{Membrane preparation}

In this paper, we have worked with the ethylcellulose (EC) magnetic membranes. The membranes, of thickness of 90-250 $\mu \mathrm{m}$ (depending on magnetic powder granulation) were prepared by solution-casting and evaporation [17, 18]. Membranes were cast from 3\% EC solution in 40:60 ethanol/toluene. The membranes with $1.5 \mathrm{~g}$ of magnetic powder MQP-16-7 (Pr-Fe-Co-Nb-B alloy $)^{1}$ and granulation: $5-50 \mu \mathrm{m}$ were cast in external field of a coil (stable magnetic field with range of induction 0-40 mT). Membranes removed from Petri dish were dried in $40^{\circ} \mathrm{C}$ for at least two days and, finally, magnetized in the field magnet with magnetic induction $B$ of about $2.5 \mathrm{~T}$ before any further analysis. Magnetic membranes with $1.0-10.5 \%$ of neodymium powder content in polymer solution and $19.1-73.0 \%$ in dry membrane were obtained. The membranes were stored in a desiccator under the vacuum conditions $(p=3 \mathrm{mmHg})$.

\section{Structure-morphology analysis of magnetic membranes}

We have used a box counting method (BCM) for calculating fractal dimension $d_{\mathrm{f}}$. BCM is one of the most common methods for calculating the fractal characteristics of a self-similar object and is described more precisely in [19-21]. For the self-similar sets, the number of nonempty coverings $N(\epsilon)$ scales with the current size of covering $\epsilon$ in the following way [19, 22]

$$
N(\epsilon) \propto \epsilon^{-d_{\mathrm{f}}},
$$

where $d_{\mathrm{f}}$ is a fractal (or box) dimension. Taking the logarithm at the limit $\epsilon \rightarrow 0$, we get

$$
d_{\mathrm{f}}=\lim _{\epsilon \rightarrow 0} \frac{\ln N(\epsilon)}{\ln 1 / \epsilon} .
$$

The above formulas are used to the image analysis of a sufficiently large section of the membrane. Images were acquired using the optical microscopy

\footnotetext{
${ }^{1}$ For simplicity, later in the text, we use MQP-16 to represent MQP-16-7.
} 
Leica DM2500M. Figure 1 shows the image of membranes with magnetic powder MQP-16 (granulation $5 \mu \mathrm{m}$ ) of amount of $1.5 \mathrm{~g}$. Texture of membrane with magnetic powder was observed with $1350 \times$ magnification.

We assumed that morphology plane and across membrane is the same. We studied a random walk across the membrane.
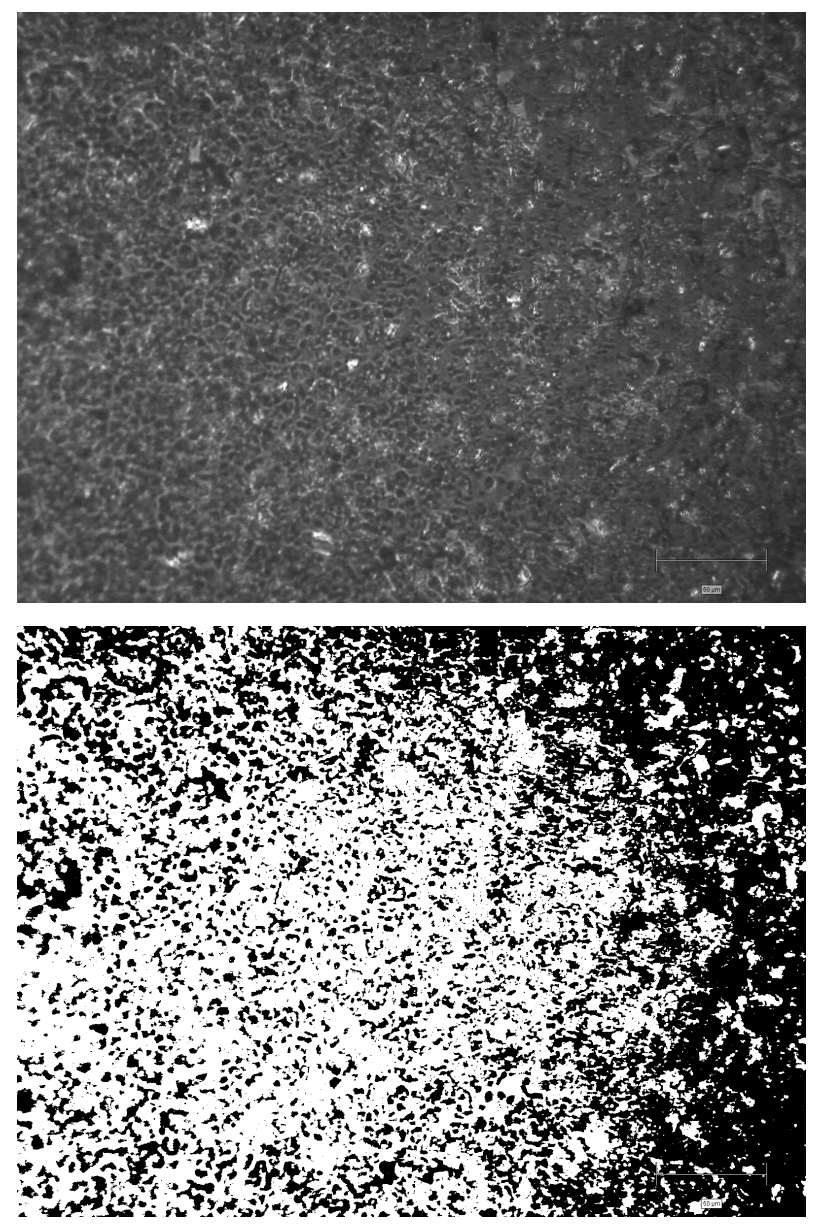

Fig. 1. Typical structure for ethylocellulose membrane with magnetic powder MQP-16 ( $5 \mu \mathrm{m})$ (top) from optical microscopy, (bottom) digitized image, as required for the fractal analyses, i.e. grey-level threshold techniques provide binary image. 


\section{Fractal dimension of random walk}

Random walk simulation were used to determine the $\alpha$ coefficient - the scaling factor between mean square displacement of molecules diffusing in modelled membranes and time. According to relation (1)

$$
\ln \left\langle r^{2}(t)\right\rangle \sim \alpha \ln t .
$$

In this specific case, the $\langle\cdot\rangle$ denotes averaging over 100 independent path starting from randomly distributed membrane points. Successive steps along a trajectory were generated as follows:

(i) a vector of a fixed length $d$ and orientation given by uniform probability distribution over $(0,2 \pi]$ was selected randomly. The vector added to the last trajectory component points a new virtual point;

(ii) if the virtual point belonged to the polymeric and magnetic part of membrane, it was added to the path;

(iii) otherwise the virtual point was abandoned.

Due to the scale-free character of relation (11), the choice of units for measuring distance and time is irrelevant. On the other hand, the simulations were performed using digitised image of a membrane and the digitisation process introduces the intrinsic scale connected with a size of a pixel. Therefore, the value of $d$ should be comparable with this size to avoid the trajectory jumping over an obstacles (large $d$ ) or not noticing them at all (small $d$ ) during the simulation. For this reason, $d=0.5$ [px] were used in our simulations. The time was measured as a number of steps along a path. The simulation

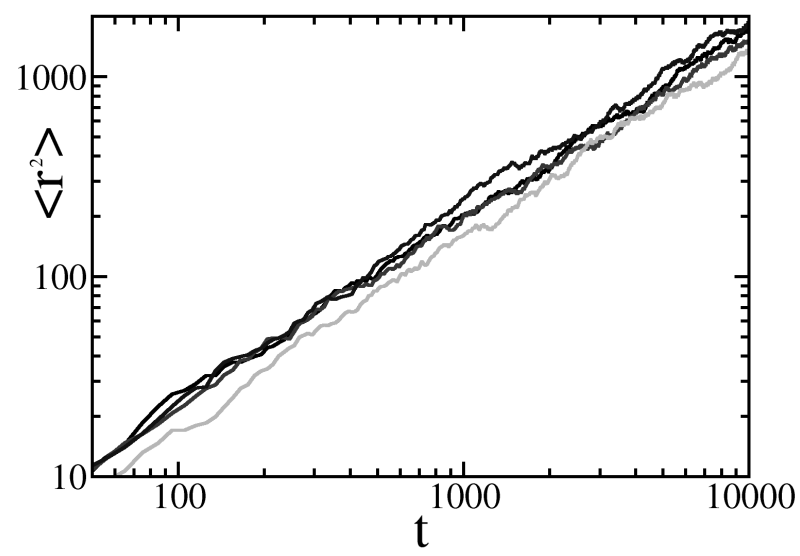

Fig. 2. Exemplary mean square displacements. 
time assured at least $10^{4}$ points in each trajectory. An exemplary mean square displacements dependence on time is presented in figure 2. Slope of the lines corresponds to the exponent $\alpha$ in relation (11).

\section{Results and discussion}

The parameters required for diffusive transport description, like anomalous exponent $\alpha$, fractal dimension of random walk $d_{\mathrm{w}}$ and fractal dimension of structure $d_{\mathrm{f}}$, are collected in Table I. The table covers membranes made of ethylcellulose with $1.5 \mathrm{~g}$ of magnetic powder, it collects also information of its granulation.

TABLE I

Fractal characteristics: fractal dimension $d_{\mathrm{f}}$, fractal dimension of random walk $d_{\mathrm{w}}$ and anomalous diffusion exponent $\alpha$, for ethylcellulose membranes with various granulation of magnetic powder. Amount of magnetic powder $1.5 \mathrm{~g}$.

\begin{tabular}{lccc}
\hline \hline Type of membrane & $\alpha$ & $d_{\mathrm{w}}$ & $d_{\mathrm{f}}$ \\
\hline MQP-16 $(5 \mu \mathrm{m})$ & $0.86 \pm 0.02$ & $2.32 \pm 0.01$ & $1.85 \pm 0.01$ \\
MQP-16 $(5 \mu \mathrm{m})$ & $0.84 \pm 0.01$ & $2.38 \pm 0.01$ & $1.86 \pm 0.01$ \\
MQP-16 $(5 \mu \mathrm{m})$ & $0.84 \pm 0.01$ & $2.38 \pm 0.01$ & $1.86 \pm 0.01$ \\
MQP-16 $(5 \mu \mathrm{m})$ & $0.84 \pm 0.01$ & $2.38 \pm 0.01$ & $1.88 \pm 0.01$ \\
MQP-16 $(<20 \mu \mathrm{m})$ & $0.87 \pm 0.01$ & $2.30 \pm 0.01$ & $1.90 \pm 0.01$ \\
MQP-16 $(<20 \mu \mathrm{m})$ & $0.87 \pm 0.01$ & $2.30 \pm 0.01$ & $1.91 \pm 0.01$ \\
MQP-16 $(20-50 \mu \mathrm{m})$ & $0.92 \pm 0.02$ & $2.17 \pm 0.01$ & $1.93 \pm 0.01$
\end{tabular}

As can be seen from figure 3, an increase of powder granulation increases the value of alpha, which approaches 0.92 , and the process is getting closer to normal diffusion. Decrease in magnetic powder granulation gives the opportunity to observe subdiffusion, where the particles of gas, transported through the membrane awaiting execution to the next move (local immobilization due to the magnetic field interaction), while in the case of normal diffusion, such molecule can make a move at any time. Mobility of gas particles is limited by the existing obstacles in the polymer matrix, in the form of magnetic powder. With the increase of powder granulation, an increase of $d_{\mathrm{f}}$ and decrease of $d_{\mathrm{w}}$ values is observed.

Based on the data from Table I, figures $4(\mathrm{~A})-(\mathrm{C})$ are plotted, i.e. probability density $P(r, t) / A^{*}$ is plotted versus $r$ for times $t=0.15,0.25,0.5,1$ and for membranes with magnetic powder granulation $(\mathrm{A})(<5 \mu \mathrm{m}),(\mathrm{B})$ $(5-20 \mu \mathrm{m}),(\mathrm{C})(>20 \mu \mathrm{m})$. As can be seen from aforementioned figures, the probability density is monotonically decreasing with $r$. The spreading of the probability in time is slower than in the case of normal diffusion (see Fig. 5). 


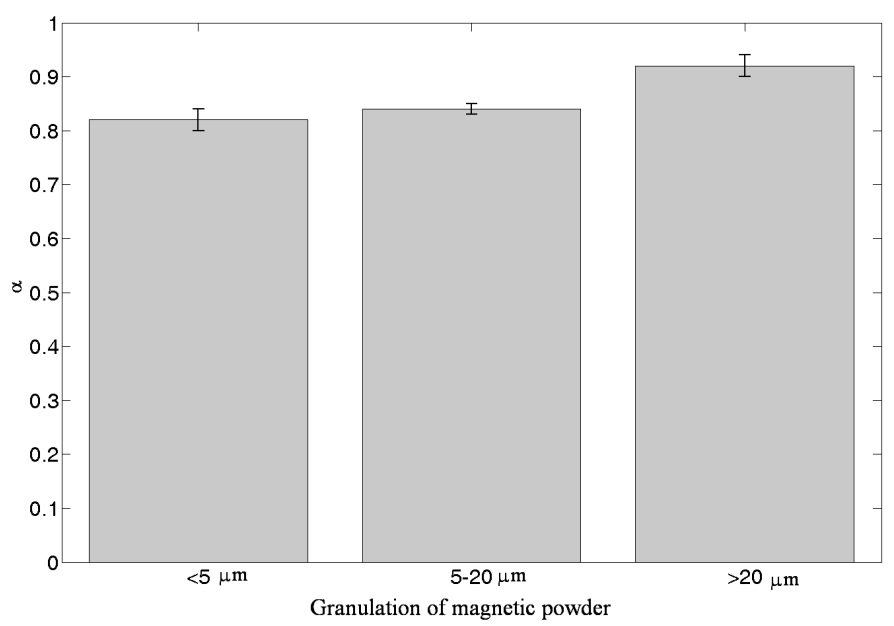

Fig. 3. Comparison of alpha values for the various magnetic powder granulation.

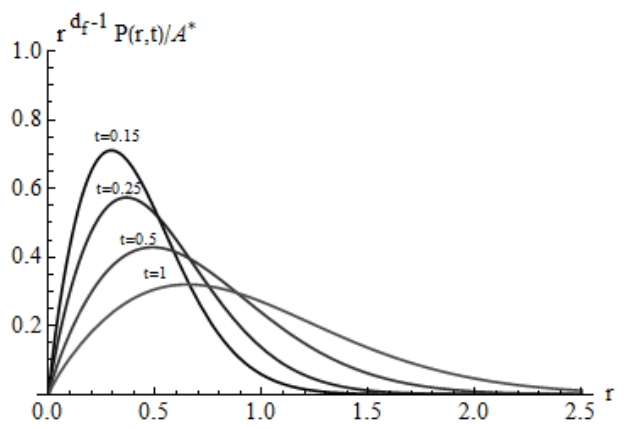

(A)

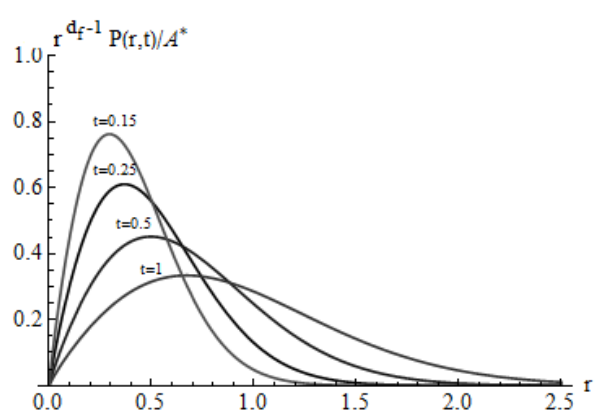

(B)

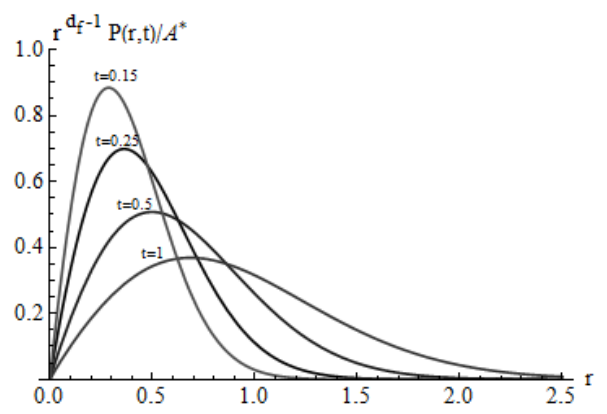

(C)

Fig. 4. Probability density at times $t=0.15,0.25,0.5,1$ for membranes with dispersed magnetic powder (A) MQP-16 $(<5 \mu \mathrm{m}), d_{\mathrm{f}}=1.86, d_{\mathrm{w}}=2.38(\mathrm{~B}) \mathrm{MQP}-16$ $(5-20 \mu \mathrm{m}) d_{\mathrm{f}}=1.90, d_{\mathrm{w}}=2.30(\mathrm{C}) \mathrm{MQP}-16(>20 \mu \mathrm{m}) d_{\mathrm{f}}=1.93, d_{\mathrm{w}}=2.17$. 


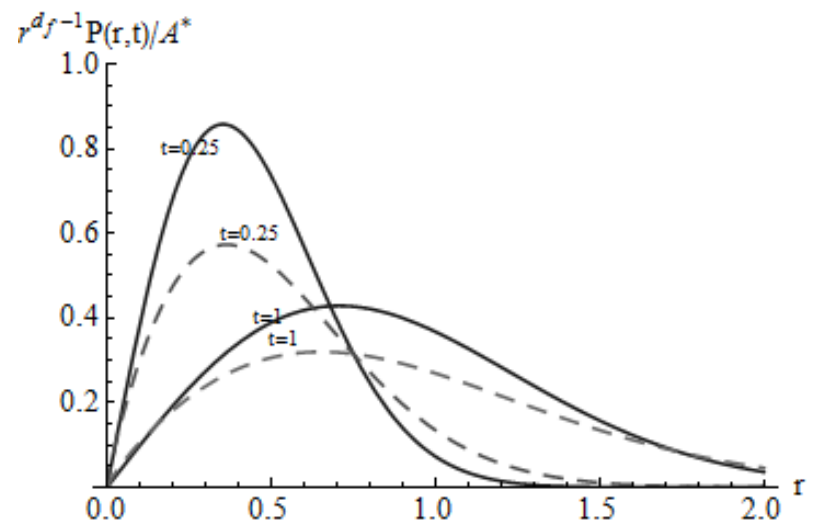

Fig. 5. Comparison of the spreading of the probability density for normal diffusion (solid line) and for the case from figure 4 (A) (dashed line).

The phenomenological diffusion equation (6) for $d_{\mathrm{f}}=1.83$ and $d_{\mathrm{w}}=2.06$ i.e. for membranes with magnetic powder MQP-B (granulation $<5 \mu \mathrm{m}$ ), has a form

$$
\frac{\partial^{0.97} P(r, t)}{\partial t^{0.97}}=\frac{1}{r^{0.78}} \frac{\partial}{\partial r}\left(r^{0.78} \frac{\partial P(r, t)}{\partial r}\right)
$$

and according to (7) it leads to

$$
\begin{aligned}
P(r, t)= & A^{*} 0.97 t^{-0.89}\left[\sum_{j=0}^{\infty} \frac{\Gamma(-0.11-j)}{\Gamma(0.11-0.97(0.11+j))}\right. \\
& \times \frac{(-1)^{j}}{j !}\left(\frac{r^{2.06}}{t}\right)^{0.97(0.11+j)} \\
& \left.+\sum_{j=0}^{\infty} \frac{\Gamma(0.11-j)}{\Gamma(0.11-0.97 j)} \frac{(-1)^{j}}{j !}\left(\frac{r^{2.06}}{t}\right)^{0.97 j}\right],
\end{aligned}
$$

where the constant $A^{*}$ is

$$
A^{*}=\frac{1.07}{\Gamma(1.44) \Gamma(0.915)}=1.143 \text {. }
$$

As observed, the increase of powder granulation causes increase of the probability density, i.e. the spreading of the probability density function in time for magnetic powder with smaller granulation is slower than in the case when powder granulation is larger. Most likely, the free volume for gas molecules increases with the increasing granulation and the probability density of encountering a particle of the gas in the polymer matrix also 
increases. The MQP-16 powder has diverse elemental composition, which is probably directly related to its different structure properties and influences the value of the probability density.

\section{Conclusions}

The complex systems, i.e. polymeric membrane with magnetic powder and the investigation of their structural and dynamical properties has been shown. The structure and morphology of the membrane is one of the most important information about the membrane system that should be incorporated into the transport equation. The fractal dimension $d_{\mathrm{f}}$ is responsible for the scaling of the mass the membrane consist of. Looking at the transport from that point of view, we consider the problem of random walk as a microscopic behaviour of the transported mass. This provides another measure of fractal structure called the random walk dimension $d_{\mathrm{w}}$. In summary, we observed that with increasing powder granulation the values of $d_{\mathrm{f}}$ increase and $d_{\mathrm{w}}$ decrease. The knowledge of the parameters $d_{\mathrm{f}}$ and $d_{\mathrm{w}}$ gave us the opportunity to use the generalized diffusion equation on fractal structure obtained by Metzler et al., i.e. equation (6). This equation is a generalization of the spherically symmetric diffusion equation in Euclidean spaces. Such equation can be used for modelling of diffusive drug release from polymeric microspheres. In our experiment, we observed that the spreading of the probability density function in time for membranes with magnetic powder is slower than in the case of normal diffusion. The increase of powder granulation caused the rise of the probability density function. This phenomena could be connected with an increase of a free volume in the polymer matrix, generated by higher powder granulation. Moreover, for lower granulation of the powder, the random walk has more subdiffusive character. This is probably due to the existence of the obstacles in the polymer matrix (magnetic powder) which limit the mobility of diffusing gas particles.

\section{REFERENCES}

[1] S. Havlin, D. Ben-Avraham, Adv. Phys. 36, 695 (1987).

[2] R. Metzler, W.G. Glöckle, T.F. Nonnenmacher, Physica A 211, 13 (1994).

[3] Y. Shia et al., J. Power Sources 195, 4865 (2010).

[4] Q. Zheng, B. Yu, S. Wang, L. Luo, Chem. Eng. Sci. 68, 650 (2012).

[5] P. Sysel et al., Desalin. Water Treat. 34, 211 (2011).

[6] J. Hradil et al., React. Funct. Polym. 67, 432 (2007).

[7] F. Cesarone, M. Caputo, C. Cametti, J. Membr. Sci. 250, 79 (2005).

[8] I.M. Sokolov, Soft Matter 8, 9043 (2012). 
[9] A. Strzelewicz, Z.J. Grzywna, J. Membr. Sci. 294, 60 (2007).

[10] Z.J. Grzywna, P. Borys, B. Kruczek, Sep. Sci. Technol. 46, 2427 (2011).

[11] D. Avnir, The Fractal Approach to Heterogeneous Chemistry, Surfaces, Colloids, Polymers, John Wiley and Sons, 1992.

[12] M. Krasowska et al., J. Membr. Sci. 415-416, 864 (2012).

[13] A. Rybak, M. Krasowska, A. Strzelewicz, Z.J. Grzywna, Acta Phys. Pol. B 40, 1001 (2009).

[14] B. Dybiec, E. Gudowska-Nowak, Phys. Rev. E80, 061122 (2009).

[15] R. Hilfer, Applications of Fractional Calculus in Physics, World Scientific, 2000.

[16] B. O’Shaughnessy, I. Procaccia, Phys. Rev. A32, 3073 (1985).

[17] A. Rybak, Z.J. Grzywna, W. Kaszuwara, J. Membr. Sci. 336, 79 (2009).

[18] Z.J. Grzywna, A. Rybak, A. Strzelewicz, Air Enrichment by Polymeric Magnetic Membranes, chapter in book: Membrane Gas Separation, ed. Y. Yampolskii, B. Freeman, John Wiley and Sons, 2010.

[19] Z.J. Grzywna, M. Krasowska, Ł. Ostrowski, J. Stolarczyk, Acta Phys. Pol. B 32, 424 (2001).

[20] Z.J. Grzywna, M. Krasowska, J. Stolarczyk, Acta Phys. Pol. B 34, 3681 (2003).

[21] Z.J. Grzywna, M. Krasowska, Inżynieria Materiałowa 4, 369 (2001) (in Polish).

[22] J.B. Bassingthwaighte, L.S. Liebovitch, B.J. West, Fractal Physiology, Oxford University Press, New York 1994. 